\title{
Esclavage et abolitions dans l'Océan Indien (1723 - 1860). Systèmes esclavagistes et abolitions dans les colonies de l'océan indien
}

\section{Bernard Gainot}

\section{(2) OpenEdition \\ 1 Journals \\ Édition électronique \\ URL : https://journals.openedition.org/ahrf/7293 \\ DOI : 10.4000/ahrf.7293 \\ ISSN : 1952-403X \\ Éditeur : \\ Armand Colin, Société des études robespierristes}

\section{Édition imprimée}

Date de publication : 1 septembre 2006

Pagination : 195-198

ISSN : 0003-4436

\section{Référence électronique}

Bernard Gainot, «Esclavage et abolitions dans I'Océan Indien (1723 - 1860). Systèmes esclavagistes et abolitions dans les colonies de l'océan indien ", Annales historiques de la Révolution française [En ligne], 345 | juillet-septembre 2006, mis en ligne le 08 juillet 2008, consulté le 01 juillet 2021. URL http://journals.openedition.org/ahrf/7293; DOI : https://doi.org/10.4000/ahrf.7293

Ce document a été généré automatiquement le 1 juillet 2021.

Tous droits réservés 


\title{
Esclavage et abolitions dans l'Océan Indien (1723 - 1860). Systèmes esclavagistes et abolitions dans les colonies de l'océan indien
}

\author{
Bernard Gainot
}

\section{RÉFÉRENCE}

Edmond Maestri (dir.), Esclavage et abolitions dans l'Océan Indien (1723 - 1860). Systèmes esclavagistes et abolitions dans les colonies de l'Océan Indien, Paris, L'Harmattan et Université de la Réunion, 2002, 451 p., ISBN 2-7475-3017-5, $38 €$.

1 Ce volume réunit trente-deux contributions au colloque organisé à Saint-Denis de la Réunion, par l'Université de la Réunion. La période couverte va de l'introduction du «Code noir" à la Réunion en 1723 jusqu'aux abolitions du XIX ${ }^{e}$ siècle et leurs conséquences.

2 En avant-propos, en quelque sorte, «L'analyse du Code noir » par Laurent Sermet reprend largement l'interprétation de Sala-Molins : interprétation raciale, qui part du postulat de la domination de la race blanche sur la race noire, et reprise de l'affirmation selon laquelle l'ordonnance de Colbert ne doit rien au droit romain 俤- affirmation rapide, qui ne mentionne pas les nombreuses études qui contestent cette version, et toutes les modifications subies par le texte original au cours de l'évolution historique.

3 La dernière contribution, celle de Ho Hai Qang, qui est un essai de formalisation des systèmes économiques, prend également la date de 1723 comme point de départ d'un «mode de production esclavagiste » qui couvre l'essentiel du XVIII siècle et du début du XIX ${ }^{e}$ siècle, jusqu'aux mutations induites à partir des années 1810 par l'interdiction de la traite et le développement de l'économie industrielle sucrière. Cette formalisation ne rend pas compte des dynamiques internes à l'espace régional considéré, de la façon 
dont les circuits européens de traite, notamment, s'insèrent, et récupèrent à leur profit des circuits préexistants de ventes d'esclaves organisés sur la côte orientale de l'Afrique, ou sur les côtes du continent indien. Il donne l'impression réductrice d'une rationalité externe aux société locales, mercantiliste ou capitaliste, faisant fi des mécanismes d'échanges pluri-séculaires propres à cette aire géographique.

Il est toujours difficile de poser abruptement une date de rupture, qui marque une détermination institutionnelle, ou économique, pour des rapports sociaux complexes, produits d'une longue évolution historique. Les rapports esclavagistes n'avaient pas disparu depuis l'Antiquité, pour réapparaître dans le sillage de la mondialisation des échanges impulsée par les économies européennes. Ces réserves émises, il faut bien proposer un découpage, et admettre qu'à partir du début du XVIII ${ }^{e}$ siècle, s'est produit un changement d'échelle, qui façonne l'identité des formations sociales en question jusqu'à nos jours.

5 Cette identité, l'article de Sudel Fuma et Jean Poirier (La mémoire de l'esclavage. De l'ethno-histoire à l'anthropologie: pour de nouveaux concepts) cherche à l'appréhender à travers l'analyse du contexte culturel de l'esclavage, aussi bien par l'étude du discours (chrétien particulièrement) qui a justifié l'esclavage, que par l'acculturation subie par la masse servile. Identité faite de déracinement par rapport aux sociétés africaines, mais aussi de constitution de nouveaux rapports sociaux qui vont révéler toute leur dynamique après l'abolition.

6 Claude Prud'homme traite précisément des rapports entre l'Église catholique et l'esclavage. L'Église a élaboré un discours visant à légitimer l'esclavage, sous réserve de quelques aménagements dans le domaine moral et humanitaire. Ce discours fait des emprunts, aussi bien au droit canon qu'au droit naturel, et se nourrit de la défiance envers la prohibition de la traite, dont l'Angleterre se fait la championne, et de la défiance envers la tradition abolitionniste liée à la Révolution française. En 1839, une rupture majeure se produit avec l'encyclique In supremo apostolatus du pape Grégoire XVI. Sans exagérer la portée de cette encyclique, on peut remarquer qu'elle manifestait une ouverture prudente en direction des arguments abolitionnistes, ouverture radicalisée par une minorité de prêtres, qui accepteront avec enthousiasme les mesures de 1848. Mais cette évolution se fait par adaptation, et non par révision d'une tradition doctrinale, intangible jusqu'à une date récente.

7 Sidi Ainouddine, dans deux articles, retrace la place de l'esclavage dans l'archipel des Comores. Il rappelle l'évolution historique de l'archipel, au croisement de l'Afrique orientale et de la péninsule arabique. L'esclavage fut généralisé par les grandes familles commerçantes arabes. Au début du $\mathrm{XIX}^{\mathrm{e}}$ siècle, les raids malgaches transformèrent plusieurs des îles en entrepôts d'esclaves principalement destinés aux Mascareignes. Le système esclavagiste vit son plein épanouissement au cours du XIX ${ }^{\mathrm{e}}$ siècle, nourri par le développement de l'économie de plantation dans l'archipel, et par la demande des planteurs des Mascareignes. Les autorités coloniales, anglaises et françaises, couvraient le trafic, avec la complicité des planteurs européens et des sultans locaux. L'étude minutieuse des conditions de vie et de travail des engagés montre que l'habillage juridique du nouveau système ne faisait que prolonger les rapports sociaux esclavagistes. Le recrutement des esclaves aux Comores a continué jusqu'en 1904, avec l'abolition décrétée à Ngazidja, vaste entrepôt de redistribution de main-d'œuvre pour toute la région. 
Cette évolution est tout à fait parallèle à celle qui se déroule sur le continent. Michel Polényk présente l'évolution de Zanzibar, sultanat arabe indépendant entre 1807 et 1888 , et vaste entrepôt d'esclaves de toute l'Afrique orientale. Le sultanat avait établi des relais vers l'Afrique des grands lacs et le Congo, d'où il retirait de précieuses cargaisons d'ivoire et d'esclaves. Ces derniers étaient à la fois objets de commerce, et vecteurs du même commerce, qui se pratiquait par caravanes de porteurs, où les conditions de charge étaient très dures. Les esclaves africains étaient ensuite vendus à des notables locaux du sultanat, ou bien expédiés vers le Golfe persique, l'Inde, le Proche-Orient. Les puissances européennes (essentiellement les Britanniques et les Allemands) étaient alors engagées dans une vaste croisade de répression de la traite, sous couvert de laquelle s'opérait le partage colonial des territoires d'Afrique orientale. Mais l'auteur montre bien que le consulat britannique à Zanzibar savait fermer les yeux sur les réalités du trafic d'hommes, lorsque ses intérêts étaient en jeu. Du moins jusqu'à l'abolition officielle de l'esclavage en 1897, qui marque un effondrement brutal de la prospérité de Zanzibar, alors intégré à l'Empire colonial allemand. Un trafic clandestin n'allait pas moins persister jusqu'au début du XX $\mathrm{X}^{\mathrm{e}}$ siècle.

9 Issa Asgarally dresse un inventaire de toutes les formes de résistance à l'esclavage, depuis les vols jusqu'aux insurrections (Systèmes esclavagistes et abolitions dans les colonies de l'océan indien -1723-1860). Vaste panorama que cherche à appréhender de façon plus précise Claude Wanquet, à travers l'étude de treize cartons des Archives nationales de Maurice, qui sont les archives des juridictions criminelles de l'île-de-France, de 1793 à 1801. Cette recherche exhaustive est d'autant plus précieuse que l'auteur signale que l'état de ces cartons se détériore gravement, jusqu'à rendre quasiment inutilisable les sources du début de l'époque consulaire. À travers les quelque soixante affaires ainsi recensées, il relève la criminalité des maitres envers les esclaves, mais surtout la répression de la criminalité de ces derniers, depuis les affaires de vols, très nombreuses pour l'an V jusqu'aux affaires de grand marronnage en bandes, pouvant aller jusqu'à l'assassinat, en passant par les affaires d'empoisonnement, qui sont, comme aux Antilles, une hantise des sociétés esclavagistes. Il note un durcissement de la justice coloniale, avec la réactivation de châtiments réprouvés par la législation criminelle révolutionnaire, comme la roue ou le bûcher. Il y voit un indice de la grande peur des colons face à la menace d'extension du décret d'abolition de l'esclavage de la Convention, dont ils cherchèrent à se préserver par l'émeute de l'an IV contre les envoyés du Directoire.

10 En 1810, les îles de France (Maurice) et Bourbon (la Réunion) sont occupées par les Anglais. En 1815, l'île Bourbon est rétrocédée à la France, tandis que l'île Maurice reste sous administration britannique. En 1835, une décision d'émancipation graduelle des 56 693 esclaves est prise ; après une période d'apprentissage, l'émancipation est effective en 1839. Vijayalakshami Teelock explore les archives d'une institution originale, le protector of slaves, qui fonctionne à Maurice, de 1829 à 1835. L'auteur cherche, à travers les doléances parvenues au Protecteur, à décrire la vie quotidienne des esclaves dans cette période de transition. Prosper Eve cherche, pour sa part, à mesurer l'effet de cette transition sur l'île voisine de Bourbon. Deux attitudes se dégagent; un refus massif de l'abolition chez les planteurs, qui met en avant le sort misérable des affranchis de Maurice, privés de cadres et de ressources. Une position minoritaire de certains administrateurs voit au contraire dans une abolition immédiate la seule voie pour conserver les colonies et pour éviter une révolte massive des esclaves. L'exemple de 
Maurice montre, qu'après une phase d'adaptation, la prospérité de la colonie peut repartir, en ayant massivement recours à des engagés asiatiques, qui ont commencé d'arriver aux Mascareignes en 1829.

11 La mise en place de l'industrie sucrière à l'île Bourbon, à partir de 1810, n'est pas restée sans effet sur l'esclavage. Une catégorie d'« esclaves techniciens » se développe. JeanFrançois Géraud présente les contours d'un échantillon de 2569 esclaves employés dans 155 usines en activité en 1834. La répartition ethnique montre que les esclaves techniciens sont très majoritairement des "cafres ", récemment importés d'Afrique de l'Est. Le travail devient plus technique, plus complexe, ce qui suppose une forme d'apprentissage et de qualification. Dans une société restée foncièrement esclavagiste, certains esclaves ont pu connaître une promotion qui a fait reculer les blocages mentaux qui confinaient l'esclave dans un statut d'infériorité, celui des "nègres de pioche" de la production traditionnelle. Mais les esclaves eux-mêmes n'ont pas forcément vécu cette qualification technique comme un progrès, car la mécanisation a aggravé leurs conditions de travail. Le travail à l'usine est encore plus aliénant que le travail de la terre.

Hubert Gerbeau présente un épisode des révoltes serviles, qui n'a pratiquement pas laissé de traces écrites, le «complot » ou l'« insurrection » de Saint-Leu, en novembre 1811. Il a recours, pour ce faire, aux témoignages indirects, et notamment aux sources orales. Le contexte spécifique de la Réunion, qui vient d'être occupée par les Anglais, a favorisé la révolte. Les insurgés ont joué des rivalités franco-britanniques, et de la perte de prestige des maîtres français, qui viennent de subir une défaite. Mais, surtout, a joué la peur du précédent de Saint-Domingue ; les colons vivent dans la hantise de voir les masses serviles suivre l'exemple de la jeune république de Haïti. Cette peur est accentuée dans le contexte démographique spécifique de Saint-Leu, où le rapport interethnique est particulièrement défavorable aux blancs. La répression, menée à partir de la mobilisation des ateliers restés fidèles, puis formalisée par les autorités britanniques, fut à la mesure de cette peur. La moitié des 300 révoltés y laissèrent la vie, et il y eut trente condamnations à mort.

13 L'exemple de Saint-Domingue est également présent dans l'analyse, menée par JeanPierre Tardieu, du débat mené à Cuba entre les réformateurs de l'économie et de la société de l'île, et les partisans du système esclavagiste, des années 1810 aux années 1850. Les premiers font valoir les avantages que la substitution du travail libre au travail servile pourrait apporter à la production de l'île. Mais ils soulignent également les dangers inhérents à la présence des masses serviles noires travaillées par les soulèvements révolutionnaires dans les autres colonies. Les esclavagistes répondent par les arguments classiques de la supériorité de la condition des esclaves, par rapport à la sauvagerie africaine, d'une part, à la misère du prolétariat européen, d'autre part. L'argument nouveau est la dénonciation de l'hypocrisie anglaise, dont la lutte contre la traite est une arme pour ruiner la production antillaise au profit de leurs possessions indiennes. Mais les différends entre les deux courants repose sur un mépris identique de la « barbarie » africaine.

14 D'autres contributions portent plus particulièrement sur l'abolition de 1848 à la Réunion (unique abolition puisque l'île avait refusé le décret révolutionnaire de 1794), et sur ses conséquences sociales. Elles illustrent toute la richesse de ce gros volume, qui apporte une contribution majeure à la connaissance historique d'un espace régional, 
qu'il est essentiel de prendre en compte dans les perspectives, actuellement renouvelées, de la world history. 